\title{
Holz und Holzprodukte in vergleichenden Ökobilanzen
}

\author{
K. Richter, H. Gugerli
}

Mit Ökobilanzen lassen sich wichtige Kenndaten zu den mit der Funktion eines Produktes über dessen gesamten Lebenszyklus zusammenhängenden Umweltbeeinflussungen ermitteln. Sie werden eingesetzt, um umweltwirksame Verbesserungen in Einzelprozessen zu erkennen respektive umzusetzen und um unter funktionsgleichen Produkten das ökologisch verträglichste auszuwählen. Für die Durchführung von vergleichenden Ökobilanzen ist es notwendig, die derzeit im Rahmen von ISO diskutierten Vorgaben und Grundsätze zu beachten, um den Anforderungen bezüglich Transparenz, Vollständigkeit, Nachvollziehbarkeit und Konsistenz zu genügen. Wegen der bedeutenden Umweltwirkungen, die von Bauvorhaben und Bautätigkeiten ausgehen, werden seit einigen Jahren auch Baumaterialien und Bauprodukte in Ökobilanzen untersucht. Dabei nehmen Holzprodukte und Holzbauteile eine Sonderstellung ein, weil sie aus einem Rohstoff hergestellt werden, der selbst integrierter Bestandteil des Ökosystems Wald ist. Mit Ausnahme der $\mathrm{CO}_{2}$-Speicherung ist es bisher allerdings nicht gelungen, die Umweltleistungen des Waldes soweit numerisch aufzubereiten, daß sie in Ökobilanzen mitberücksichtigt werden. Dennoch zeigen die ausgewählten Beispiele aus den Anwendungsbereichen Hochbaukonstruktionen und Stromleitungsbau, daß Holzprodukte in vergleichenden Gegenüberstellungen zu alternativen Materialien ökologisch gut abschneiden, insbesondere aufgrund des geringen Verbrauchs fossiler Energieträger, eines geringen Beitrags zum Treibhauseffekt und ein geringes Abfallvolumen.

\section{Wood and timber derived products in comparative Life Cycle Assessment studies}

Life Cycle Assessment (LCA) aspire to quantify the potential environmental impacts of a product system throughout its life cycle. The technique is used to identify and realize opportunities to improve the environmental aspects of processes, and to compare between products with equivalent function and performance in order to select the environmentally best ones. Especially when the intended application is a comparative assertion, it is inevitable that the key features and methodological framework currently discussed in ISO be accepted and applied to fulfill the specific requirements of transparency, completeness, reproducibility, reliability, and consistency. Because construction affects our environment to a significant extent, building materials and products have been evaluated in LCA studies for several years. In this context, wood and timber based products do have an exceptional position, in that they are produced from a resource which itself is an integral part of the forest ecosystem. It has not yet been possible to

\section{K. Richter}

EMPA Dübendorf, Abteilung Holz, CH-8600 Dübendorf

H. Gugerli

INTEP AG, Lindenstr. 34, CH-8034 Zürich quantify the environmental benefits of sustainable forestry to such an extent that they can be used in LCA to underline the environmental benefits of wood with $\mathrm{CO}_{2}$-storage being the exception to the rule. Nevertheless, the examples selected from applications in building construction and power supply lines manifest that timber derived products have an excellent environmental position when compared to alternative products. This is mainly due to the sparing use of fossile energy resources, low contribution to the greenhouse effect, and small waste volume.

\section{1}

\section{Einleitung}

Ökobilanzen haben sich in den letzten Jahren zu einem wichtigen Instrument des Umweltmanagements entwickelt. Produktbezogene Ökobilanzen zielen darauf ab, aus den über den gesamten Lebensweg eines Produktes ermittelten Stoff- und Energieflüssen die Umweltwirkungen zu ermitteln und zu bewerten. Dadurch wird es möglich, umweltbelastende Prozesse zu erkennen, in ihren Wirkungen zu quantifizieren und gegebenenfalls Massnahmen zur Reduktion der Umweltbelastung abzuleiten (Optimierungsfunktion). Werden mehrere Produkte mit vergleichbaren Funktionen und Eigenschaften analysiert, so können aus Ökobilanzen Entscheidungsgrundlagen für eine umweltverträgliche Produktauswahl abgeleitet werden (Vergleichsfunktion). Schließlich können die aufbereiteten Sachinformationen aus den Stoff- und Energieflüssen als Grundlage zur Information von Kunden, Umweltverbänden und Behörden (Kommunikationsfunktion) dienen.

Die Möglichkeiten des Einsatzes der Energie- und Stofflußbzw. Ökobilanzmethodik zur Optimierung von Prozessen und betrieblichen Abläufen auf Unternehmensebene sind u.a. von Braunschweig und Müller-Wenk 1993 sowie Gilgen et al. 1993 aufgezeigt worden. Unternehmungen verschiedenster Branchen setzen die Methoden ein und berichten in ihren Umwelt- und Jahresberichten von den Ergebnissen der betriebsinternen Ökobilanzierung (z.B. Isover 1994, Flumroc 1995). Ein sehr viel größeres Gewicht, gemessen an der Zahl der veröffentlichten Studien, wird allerdings den produktbezogenen Ökobilanzen beigemessen. Rubik et al. (1995) berichten von über 270 Produktbilanzen, die zwischen 1974 und 1994 im deutsch- und englischsprachigen Raum veröffentlicht wurden. Sie verweisen darauf, daß es darüber hinaus noch eine weitere Anzahl abgeschlossener, aber nicht öffentlich zugänglicher Studien gibt. Die zeitliche Verteilung der Veröffentlichungen läßt eine deutliche Häufung in den letzten sieben Jahren erkennen. Interessant ist die Tatsache, daß knapp die Hälfte der Studien von Autoren aus Deutschland (ca. 90) und der Schweiz (ca. 60) erstellt wurden.

Während in der Anfangsphase der Ökobilanzierung Produkte aus dem Verpackungsbereich mit Abstand am meisten untersucht wurden, werden seit einigen Jahren auch Baumaterialien in Ökobilanzen beurteilt. Im Gegensatz zu 
Konsumgütern mit ihren kurzen Umlaufzeiten ist bei Investitionsgütern durch die lange Nutzungsphase eine Lebenszykluserfassung mit besonderen Problemen verbunden (Richter 1994), so daß in vielen Studien und vergleichenden Gegenüberstellungen meist nur die Lebenszyklusphasen bis zur Produktion quantitativ bewertet sind.

Voraussetzung für eine Baumaterialbewertung sind Dateninventare über die herstellungsbezogenen Stoff- und Energieflüsse. Erste umfassende Daten hat Kohler 1986 publiziert und im Rahmen seiner Arbeiten periodisch aktualisiert (Kohler 1994). Jüngste Daten zur grauen Energie von Baustoffen haben Kasser et al. (1995) zusammengetragen; umfassende, aus stofflichen Ein- und Austrägen abgeleitete Ökoinventare und Wirkungsbilanzen von Baumaterialien haben Weibel und Stritz (1995) berechnet.

In diese Datensammlungen sind auch Sachbilanzen zu Holzprodukten eingeflossen, die seit Beginn der neunziger Jahre an der Abteilung Holz der EMPA erhoben und laufend an die aktuellen Erkenntnisse angepaßt werden (Richter, Sell 1992, Richter 1995). Sie sind als Ergänzung und Erweiterung der frühen Arbeiten zur energetischen Beurteilung von Holzprodukten und Herstellungsverfahren von Holzwerkstoffen zu sehen (Corrim 1976, Ressel 1986).

Dem Bedürfnis vieler Architekten, Planer und auch Bauherren entsprechend werden die Angaben aus den Dateninventaren vermehrt zur materialvergleichenden Gegenüberstellung für eine umweltoptimierte Baustoff- bzw. Bauprodukteauswahl eingesetzt. Im nachfolgenden Beitrag werden die dabei zu beachtenden Rahmenbedingungen sowie wichtige Beurteilungsparameter vorgestellt und anhand von zwei Beispielen gezeigt, welche Stellung Holzprodukte bei derartigen vergleichenden Gegenüberstellungen einnehmen.

\section{2}

\section{Aufbau einer Ökobilanz}

Methodisch läßt sich eine Ökobilanz in vier Teilschritte 'Zieldefinition, Sachbilanz, Wirkungsbilanz und Bilanzbewertung' gliedern, die auch das Gerüst für das im ISO angestrebte Normenwerk bilden.

Der Zieldefinition und Systemabgrenzung kommt innerhalb der Studien eine Schlüsselrolle zu. Hier wird festgelegt, welche Produkte mit welchen funktionalen Eigenschaften unter welchen Bedingungen untersucht werden. Die Bearbeiter einer Ökobilanz haben prinzipiell einen beträchtlichen Ermessensspielraum, da der hohen Komplexität der Prozeß-Umwelt Beziehungen oft nur durch Vereinfachungen, das Setzen von Randbedingungen oder der Vernachlässigung weniger wichtiger Zusammenhänge begegnet werden kann. Die hierbei beachteten Regeln sind in der Systemabgrenzung zu nennen. Wichtigste Grundsätze sind:

Transparenz:

Vollständigkeit:

Nachvollziehbarkeit: Es muß erkenntlich sein, wie die wiedergegebenen Daten zustandegekommen sind und sie müssen reproduzierbar sein.
Einheitlichkeit/

Neutralität/

Konsistenz:
Annahmen und Bewertungen sind anhand einheitlicher Kriterien vorzunehmen. Verglichene Produkte müssen dem gleichen

Verwendungszweck und denselben Grundfunktionen dienen (funktionale Gleichheit).

Die Ergebnisse der Stoff- und Energieanalysen der betrachteten Prozesse werden zahlenmässig im Dateninventar (Sachbilanz) abgelegt. Die Inventare bestehen meist aus einer großen Menge mehrheitlich massenbezogener Größen (energetische Verbräuche werden als stoffliche Ressourcenangaben berücksichtigt). Obwohl die Sachbilanz im Vergleich zu den anderen Bearbeitungsstufen mehrheitlich auf objektiven, meßbaren Sachverhalten aufbaut, ist auch sie nicht ohne Festlegungen und Annahmen (Entscheidungen) der Ausführenenden erstellbar, wodurch nachfolgende Bewertungen und Ergebnisse beeinflußt werden können. Das Dateninventar ist das Herzstück einer Ökobilanz. Je sorgfältiger und vollständiger die Qualität der hier zusammengetragenen Daten ist, umso mehr Bedeutung erhalten die aus der Gesamtstudie abzuleitenden Erkenntnisse.

Die Zuordnung der Sachbilanzdaten auf ausgewählte Umweltwirkungen erfolgt in der Wirkungsbilanz. Sie stellt den ersten Schritt zur Bewertung der Energie- und Stoffflußdaten dar. Sie wird heute mehrheitlich nach der von Heijungs et al. (1992) vorgeschlagenen wirkungsorientierten Klassierung vorgenommen. Das Modell definiert für eine beschränkte Anzahl von Umweltbelastungen sogenannte Leitemissionen. Andere Substanzen werden in Abhängigkeit ihrer potentiellen schädigenden Wirkung auf diese Leitsubstanzen bezogen.

Daraus resultieren Gewichtungsfaktoren, die für die Ermittlung der jeweiligen Gesamtumweltbelastung herangezogen werden. Die folgenden Wirkungskategorien werden in den unter 4 dargestellten Beispielen verwendet (Höhere Zahlenwerte drücken dabei jeweils eine stärkere Umwelteinwirkung aus).

\section{- Treibhauseffekt}

Verschiedene Gase, welche alle zum global wirkenden Treibhauseffekt beitragen, werden mittels Wirkungsfaktoren (meist für den Zeithorizont 100 Jahre) auf Kohlendioxid (kg $\mathrm{CO}_{2}$ eq) umgerechnet.

- Versauerung von Boden und Gewässern

Alle für die Versauerung relevanten Luftemissionen werden über Wirkungsfaktoren auf Schwefeldioxid ( $\mathrm{kg} \mathrm{SO}_{2}$ eq) umgerechnet, indem ihr Abgabepotential von $\mathrm{H}+-$-Ionen als Referenz herangezogen wird.

\section{- Photochemische Ozonbildung}

Alle Gase, die zur Bildung von photochemischen Oxidantien beitragen, werden mittels Wirkungsfaktoren auf Ethylen ( $\mathrm{kg}$ $\mathrm{C}_{2} \mathrm{H}_{2}$ eq) umgerechnet.

\section{- Überdüngung}

Emissionen in Wasser, Boden oder Luft werden über Wirkungsfaktoren in Phosphat ( $\mathrm{kg} \mathrm{PO}_{4}$ eq.) umgerechnet, indem ihr Düngungspotential in bezug zu Phosphat als Basis angenommen wird.

\section{- Humantoxizitä}

Emissionen mit einer Auswirkung auf die menschliche Gesundheit werden mit Wirkungsfaktoren multipliziert, die von der Substanz und dem Umweltmedium abhängen (Luft, Wasser, Boden). Die einzelnen Effekte werden zu einer Äquivalenzzahl (kg Körpergewicht) addiert. Diese beschreibt, wieviel Masse 
des menschlichen Körpers in Kilogramm bis zum toxikologischen Limit exponiert ist.

\section{- Ökotoxizität}

Emissionen mit Einfluß auf die Stabilität von Ökosystemen werden mit Äquivalenzfaktoren für Wasser- und Bodenökosysteme multipliziert. Die einzelnen Effekte beschreiben das kritische Volumen (in $\mathrm{m}^{3}$ Wasser oder in $\mathrm{kg}$ Boden), das durch jede Substanz verunreinigt wird, und werden addiert zu Gesamtbelastungen für Wasser- und Bodenökotoxizität.

Als weitere Summengrößen werden angegeben, obwohl sie nicht zu den Wirkungskategorien im o.a. Sinne gehören:

\section{- Primärenergieinhalt}

Drückt den Verbrauch und Inhalt fossiler und erneuerbarer energetischer Ressourcen in Megajoule aus.

\section{- Feste Abfälle}

Die in fester Form anfallenden und zur Deponie gelangenden Abfälle werden als Gesamtmasse oder aufgeteilt nach den in den spezifischen Gesetzeswerken vorgegebenen Abfallkategorien (Inertstoff-, Reaktor-, Sonderabfalldeponie) angegeben.

Über den anschließenden Schritt der Bilanzbewertung gibt es noch keine verbreitet akzeptierten Vorgaben. Sie ist nicht mehr allein anhand objektiver Vorgaben möglich, politische, soziale, regionale Abwägungen müssen hier berücksichtigt werden.

\section{3}

\section{Holz und Holzprodukte in Ökobilanzen}

\section{1}

\section{Ökologische Grundeigenschaften}

Der Werkstoff Holz nimmt aus ökologischer Sicht eine besondere Stellung ein. Bei seiner Entstehung ist Holz selbst integrierter Bestandteil des Ökosystems Wald, ist Lebensraum für Mikroorganismen und höhere Tiere und unterliegt als Biomasse den Gesetzen des natürlichen Abbaus. Für die Überführung zum Roh- und Werkstoff sind Bearbeitungs- und Veredelungsschritte nötig, die ihrerseits Umwelteinwirkungen in Form von Emissionen auslösen, andererseits ein Baumaterial schaffen, das am Ende der Nutzung gleichwohl, und meist noch unter Energiegewinn, in die Ausgangsstoffe $\mathrm{CO}_{2}$ und $\mathrm{H}_{2} \mathrm{O}$ zurückgeführt werden kann. Damit wird $\mathrm{Holz}$ aus nachhaltig bewirtschafteten Wäldern wie kein anderer Baustoff den Anforderungen einer ökologischen Kreislaufwirtschaft gerecht.

Allerdings ist es bisher nicht gelungen, die Gesamtheit dieser 'ökologischen Qualitäten' zahlenmässig ausreichend zu untermauern und in Ökobilanzen auszudrücken. Die auch ökologisch bedeutsamen Schutz- und Wohlfahrtsfunktionen bewirtschafteter Wälder entziehen sich noch weitgehend einer ökonomischen und ökologischen Bewertung und müssen, insbesondere wenn Holzprodukte mit anderen Werkstoffen in
Ökobilanzen verglichen werden, als qualitative Faktoren berücksichtigt werden.

\section{2}

\section{Ökoinventare von Holz- und Holzprodukten}

Wird der Naturstoff Holz zum Handels- und Wirtschaftsprodukt, lassen sich die Stoffflüsse prinzipiell gleich wie bei anderen Werkstoffen erfassen. Tabelle 1 gibt einen Überblick über ausgewählte Summen- und Wirkungspotentiale von Holzprodukten (bis zur Stufe Fertigung).

In Bild 1 ist der Primärenergieinhalt auf die einzelnen Prozessschritte aufgeteilt. Die Zahlen belegen, daß bei allen Produkten der weitaus größte Teil des Energieinhaltes durch den Heizwert des Holzes verursacht ist, der laut Konvention in der Kenngröße 'Primärenergieinhalt' eingerechnet wird. Dieser kann, bei der energetischen Verwertung der Reststoffe am Ende der Nutzung, unter Berücksichtigung der Umwandlungsverluste zurückgewonnen und bei einer Lebenszyklusbetrachtung gutgeschrieben werden.

Die in Tabelle 1 negativ ausgewiesenen KohlendioxidÄquivalente sind in Bild 2 ebenfalls weiter aufgeteilt dargestellt. Es zeigt sich, daß die Kohlendioxidspeicherung im Holz die Abgaben des Gases aus Verbrennungs- und Produktionsprozessen bei weitem kompensiert. Wird das Holz am Ende seiner Nutzungsdauer verbrannt oder biologisch abgebaut,

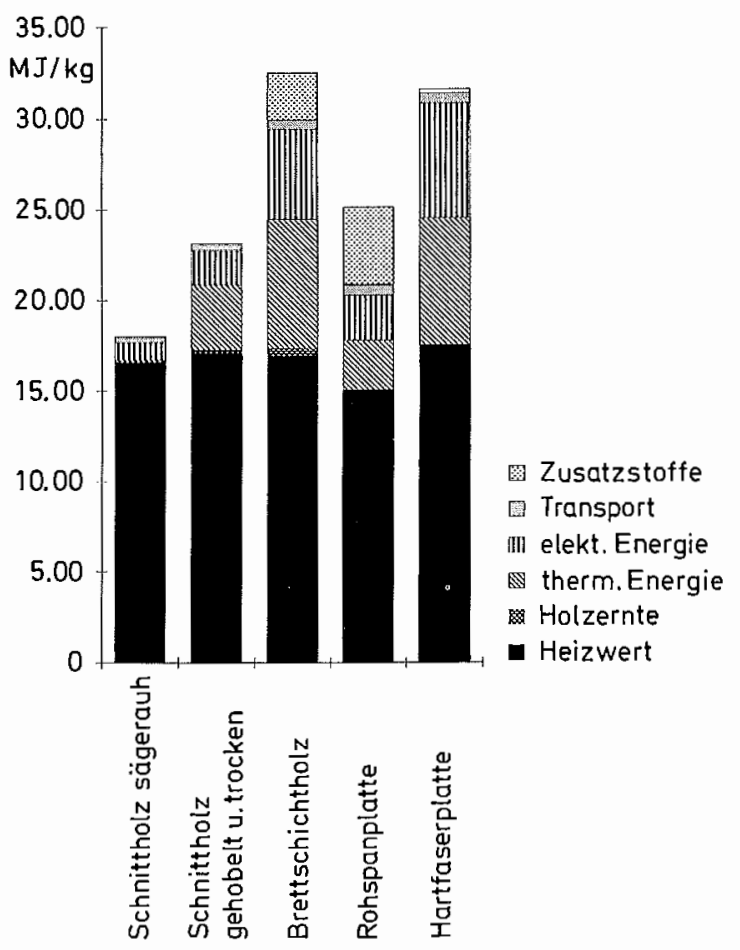

Bild 1. Kumulierter Primärenergieinhalt der Holzhalbfabrikate Fig. 1 Cumulated primary energy consumption of semi finished timber products

Table 1. Summenparameter und ausgesuchte Wirkungspotentiale pro kg bereitgestelltes Holzhalbfabrikat

\begin{tabular}{|c|c|c|c|c|c|c|}
\hline & $\begin{array}{l}\text { Primärenergieinh } \\
\text { MJ }\end{array}$ & $\begin{array}{l}\text { Treibhauseffekt } \\
\mathrm{kg} \mathrm{CO}_{2}-\mathrm{eq}\end{array}$ & $\begin{array}{l}\text { Ozonbildung } \\
\mathrm{kg} \mathrm{C}_{2} \mathrm{H}_{2} \text {-eq }\end{array}$ & $\begin{array}{l}\text { Versauerung } \\
\mathrm{kg} \mathrm{SO}_{2}-\mathrm{eq}\end{array}$ & $\begin{array}{l}\text { Inertstoff-Deponie } \\
\mathrm{kg}\end{array}$ & $\begin{array}{l}\text { Reaktor-Deponie } \\
\mathrm{kg}\end{array}$ \\
\hline Nadelschnittholz & 18.02 & -1.53 & $4.26 \mathrm{E}-08$ & $4.17 \mathrm{E}-04$ & $1.00 \mathrm{E}-02$ & $3.60 \mathrm{E}-04$ \\
\hline $\begin{array}{l}\text { Nadelschnittholz } \\
\text { gehobelt und trocken }\end{array}$ & 23.10 & -1.62 & $4.44 \mathrm{E}-08$ & $8.00 \mathrm{E}-04$ & $1.27 \mathrm{E}-02$ & $5.21 \mathrm{E}-04$ \\
\hline Brettschichtholz & 32.48 & --1.44 & $2.22 \mathrm{E}-07$ & $2.00 \mathrm{E}-03$ & $2.35 \mathrm{E}-02$ & $1.76 \mathrm{E}-03$ \\
\hline Spanplatte & 25.33 & -1.04 & $1.45 \mathrm{E}-07$ & $2.85 \mathrm{E}-03$ & 3.37E-02 & $2.75 \mathrm{E}-03$ \\
\hline Faserplatte & 31.59 & -1.47 & $5.44 \mathrm{E}-07$ & 7.27E-03 & $6.42 \mathrm{E}-02$ & $5.63 \mathrm{E}-03$ \\
\hline
\end{tabular}




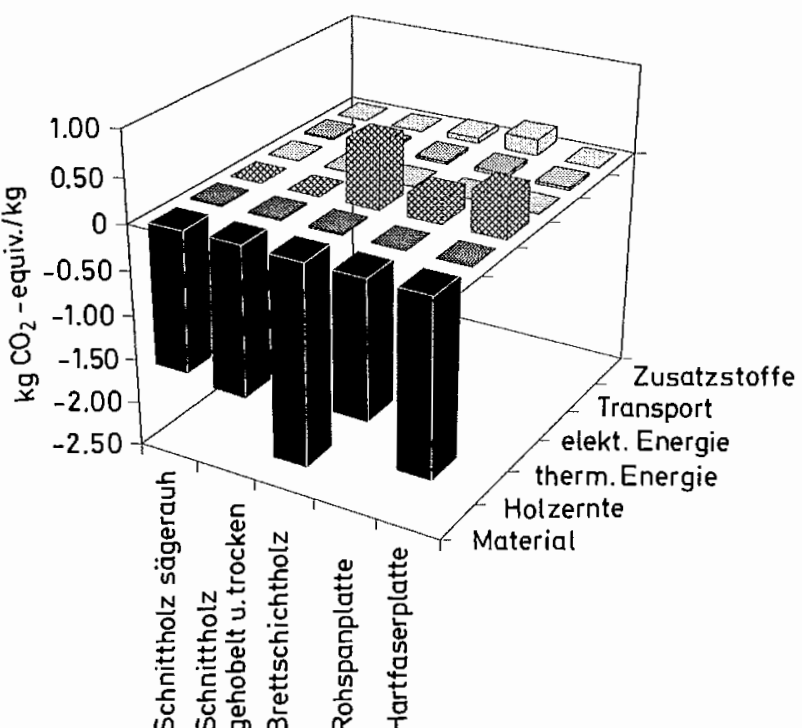

Bild 2. Kohlendioxid-Äquivalent der Holzhalbfabrikate Fig. 2. $\mathrm{CO}_{2}$-equivalent of semi finished timber products

müssen die dann freigesetzten $\mathrm{CO}_{2}$-Emissionen berücksichtigt werden. In einer Gesamtbilanz wird Holz somit als $\mathrm{CO}_{2}$-neutraler Rohstoff behandelt.

Wichtig ist die Tatsache, daß in der Wald- und Holzindustrie enge Rohstoffverknüpfungen vorliegen. Durchforstungshölzer aus der Waldpflege sind Rohstoffe der Papier- und Plattenindustrie, und auch die beim Sägen von Rundholz anfallenden Schwarten, Spreissel und Hackschnitzel werden als Kuppelprodukte in den Plattenwerken einer höheren, ökonomisch wie ökologisch sinnvollen Wertschöpfung zugeführt. Aus diesem Grund ist auch das Abfallaufkommen von Holzprodukten gegenüber anderen Werkstoffen gering.

\section{4}

\section{Vergleichende Ökobilanzen}

\section{1}

\section{Hochbaukonstruktionen nach ökologischen Gesichtspunkten}

Einen umfassenden und aktuellen Ansatz zur materialübergreifenden Bauteilbewertung stellt die SIA Dokumentation D0123 (1995) vor. Sie bietet ein Instrument für die Auswahl und Optimierung von Holzbaukonstruktionen nach ökologischen Gesichtspunkten. Für acht Bauteilkategorien des Hochbaus werden insgesamt 65 Konstruktionen aus dem Geschoßwohnungsbau gegenübergestellt, die größtenteils aus ökologisch und gestalterisch fortschrittlichen Wohnbauten stammen und die derzeitigen technischen Anforderungen bezüglich Schall, Wärme und Bautechnik in vergleichbarer Weise erfüllen.

\subsection{1}

\section{Randbedingungen}

Die Bewertung der Umweltauswirkungen erfolgt, wie in Bild 3 dargestellt, anhand von quantitativen, wissenschaftlichen Indexzahlen (für Rohstoffaufbereitung und Herstellung) und qualitativen, auf die Baupraxis ausgerichtete Profilkriterien (für die Verarbeitung auf der Baustelle, die Nutzung und die Entsorgungsphase). Die auf der Ökobilanzmethodik basierten Indexzahlen weisen den Treibhauseffekt in $\mathrm{CO}_{2}$ - $\ddot{\text { qquivalenten }}$ und die Versauerung in $\mathrm{SO}_{2}$ - $\mathrm{A} q u i v a l e n t e n$ aus. Als

Zusatzinformation werden der Primärenergieinhalt angegeben und die wichtigsten bauphysikalischen Kenngrößen, wie z.B. Masse, $\mathrm{k}$-Werte, Schalldämmwerte, genannt.
Die Qualität der einbezogenen Grundlagendaten ist nicht bei allen Materialien gleich gut, die Größenordnungen sind aber gewahrt. Im Unterschied zu den bauphysikalischen Daten können die ausgewiesenen Umweltparameter je nach Produktionsstandort, Herstellungstechnologie und Transportdistanz erheblich variieren, ein Schwankungsbereich bei den Ergebnissen von $\pm 30 \%$ ist angegeben.

Der Zeitfaktor spielt für die Einschätzung der Umwelteinflüsse durch Rüickbau-, Erneuerungs- und Entsorgungsprozesse eine wichtige Rolle, da sich mit längerer Nutzungsdauer die ökologische Rückzahldauer verbessert, Die Nutzungszeit der einzelnen Schichten wird nach den Angaben des Amts für Bundesbauten AFB (1995) berücksichtigt. Für Gebäude und deren Tragstruktur wird die Nutzungszeit von 80 Jahren berücksichtigt. Für Vergleiche zwischen den Konstruktionen sind die Index-Zahlen der entsprechenden Konstruktion pro $\mathrm{m}^{2}$ und pro Nutzungsjahr ausgewiesen.

\subsection{2}

\section{Ergebnisse}

Aus vier Bauteilkategorien sind die Ergebnisse der Indexwerte von je vier typischen Konstruktionen, davon mindestens eine in Holz, wiedergegeben (Tabelle 2). Die Indexdatenwerte beziehen sich ausschließlich auf die erste Lebenszyklusphase 'bis Fabriktor'. Im Profil werden der Aufwand für die Baustellentransporte und Verarbeitung auf der Baustelle (B), die Emissionen bei der Verarbeitung sowie

ökologisch/toxikologisch problematische Bestandteile während der Nutzung ( $R$ ), die Instandhaltung und Instandsetzung sowie Ersatz und Erneuerung $(\mathrm{N})$ und der spätere Rückbau und die Entsorgung (E) erfaßt. Die Profilkriterien werden qualitativ auf einer Skala von günstig bis ungünstig beurteilt. Tabelle 2 enthält eine Zusammenfassung (AFB 1995) der ausführlichen Profil-Beurteilungen. Das Gesamturteil ergibt sich aus den Index- und Profilinformationen.

Aufgrund der Einbausituationen wurde bei den Holzbauteilen kein chemischer Holzschutz vorgesehen, ein zusätzlicher Aufwand für die Oberflächenbehandlung Während der Nutzungsphase ist im Profil berücksichtigt. In der Kenngröße 'Primärenergie-Inhalt erneuerbar' ist der Heizwert der Materialien enthalten. Er beträgt beim Holz ca. 16 MJ/kg und kann nach dem Rückbau der Konstruktionen bei einer thermischen Verwertung mit geringen Verlusten genutzt werden. Dieser Anteil ist in der rechten Spalte der Tabelle2 ausgewiesen.

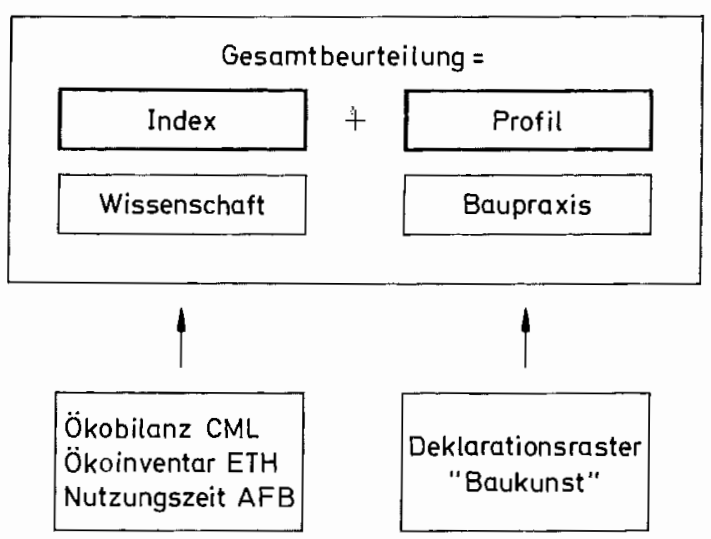

Bild 3. Komponenten zur ökologischen Beurteilung von Baukonstruktionen

Fig. 3. Components of the environmental evaluation of construction components 
Table 2. Index-Werte, Profil-Information und Primärenergieinhalt (PEI) als Zusatzinformation für Hochbaukonstruktionen (nach SIA, 1995)

\begin{tabular}{|c|c|c|c|c|c|c|c|c|c|}
\hline \multirow[t]{3}{*}{ Konstruktion } & \multirow[t]{3}{*}{$\begin{array}{l}\text { Bauteil } \\
\text { Nr. }\end{array}$} & \multicolumn{2}{|l|}{ Index } & \multicolumn{4}{|c|}{ Profil } & \multirow{2}{*}{\multicolumn{2}{|c|}{$\begin{array}{l}\text { Zusatzinformation } \\
\text { PEI MJ } / \mathrm{m} 2\end{array}$}} \\
\hline & & \multirow{2}{*}{$\begin{array}{l}\mathrm{CO}_{2} \mathrm{eq} \\
\mathrm{g} / \mathrm{m}^{2} \mathrm{a}\end{array}$} & \multirow{2}{*}{$\begin{array}{l}\mathrm{SO}_{2} \mathrm{eq} \\
\mathrm{g} / \mathrm{m}^{2} \mathrm{a}\end{array}$} & \multirow[t]{2}{*}{ B } & \multirow[t]{2}{*}{$\mathrm{R}$} & \multirow[t]{2}{*}{$\mathrm{N}$} & \multirow[t]{2}{*}{$\mathrm{E}$} & & \\
\hline & & & & & & & & $\begin{array}{l}\text { nicht } \\
\text { erneuerbar }\end{array}$ & $\begin{array}{l}\text { erneuerbar } \\
\text { (nutzbar) }\end{array}$ \\
\hline \multicolumn{10}{|l|}{ Decken/Böden } \\
\hline Beton & E0.09 & 1608 & 6.26 & 0 & + & - & + & 791 & $\begin{array}{l}27 \\
(-)\end{array}$ \\
\hline Holzbalken & $\mathrm{E} 0.12$ & 816 & 4.61 & 0 & + & + & 0 & 490 & $\begin{array}{l}1079 \\
(660)\end{array}$ \\
\hline $\begin{array}{l}\text { Holzbalken } \\
\text { Betonverbund }\end{array}$ & E0.13 & 821 & 4.86 & - & + & - & 0 & 553 & $\begin{array}{c}499 \\
(310)\end{array}$ \\
\hline Holz-Kastenelemente & E0.14 & 853 & 3.78 & - & + & + & + & 691 & $\begin{array}{l}2397 \\
(1000)\end{array}$ \\
\hline \multicolumn{10}{|l|}{ Flachdächer } \\
\hline $\begin{array}{l}\text { Beton } \\
\text { Umkehrdach }\end{array}$ & $\mathrm{E} 1.15$ & 1530 & 8.17 & + & - & + & + & 1461 & $\begin{array}{c}25 \\
(-)\end{array}$ \\
\hline $\begin{array}{l}\text { Beton } \\
\text { Bitumen }\end{array}$ & $\mathrm{E} 1.18$ & 2171 & 9.73 & 0 & + & 0 & 0 & 1540 & $\begin{array}{c}37 \\
(-)\end{array}$ \\
\hline $\begin{array}{l}\text { Holzbalken } \\
\text { Bitumen }\end{array}$ & E1.21 & 1059 & 6.61 & 0 & 0 & 0 & 0 & 982 & $\begin{array}{c}921 \\
(430)\end{array}$ \\
\hline $\begin{array}{l}\text { Holzbalken } \\
\text { PVC } \\
\text { Außenwände über Terrain }\end{array}$ & E1.23 & 1266 & 6.86 & 0 & - & 0 & 0 & 731 & $\begin{array}{c}964 \\
(466)\end{array}$ \\
\hline $\begin{array}{l}\text { Backstein } \\
\text { Faserzement }\end{array}$ & $\mathrm{E} 4.38$ & 1471 & 6.29 & 0 & + & + & + & 967 & $\begin{array}{l}134 \\
(-)\end{array}$ \\
\hline $\begin{array}{l}\text { Kalksandstein } \\
\text { zweischalig }\end{array}$ & $\mathrm{E} 4.40$ & 841 & 2.89 & - & + & 0 & + & 741 & $\begin{array}{c}37 \\
(-)\end{array}$ \\
\hline Porenbeton & $\mathrm{E} 4.46$ & 940 & 3.18 & + & + & + & + & 595 & $\begin{array}{c}15 \\
(-)\end{array}$ \\
\hline $\begin{array}{l}\text { Holzständer } \\
\text { Holzverkleidung }\end{array}$ & E4.47 & 574 & 3.10 & 0 & + & 0 & + & 493 & $\begin{array}{l}1343 \\
(650)\end{array}$ \\
\hline $\begin{array}{l}\text { Holzständer } \\
\text { Faserzement } \\
\text { Trennwände }\end{array}$ & $\mathrm{E} 4.48$ & 719 & 3.46 & 0 & 0 & + & 0 & 515 & $\begin{array}{c}819 \\
(526)\end{array}$ \\
\hline Vollgips & M1.60 & 653 & 4.23 & + & + & + & + & 422 & $\begin{array}{c}7 \\
(-)\end{array}$ \\
\hline $\begin{array}{l}\text { Holzständer } \\
\text { Gipskarton }\end{array}$ & M1.61 & 487 & 2.60 & + & + & + & - & 261 & $\begin{array}{l}194 \\
(64)\end{array}$ \\
\hline $\begin{array}{l}\text { Metallständer } \\
\text { Gipskarton }\end{array}$ & M1.62 & 694 & 3.84 & + & + & + & - & 403 & $\begin{array}{c}99 \\
(-)\end{array}$ \\
\hline $\begin{array}{l}\text { Metallständer } \\
\text { Vollgipsplatten }\end{array}$ & M1.63 & 631 & 3.60 & + & + & + & + & 392 & $\begin{array}{c}10 \\
(-)\end{array}$ \\
\hline $\begin{array}{l}\text { Backstein } \\
\text { verputzt }\end{array}$ & M1.65 & 872 & 3.30 & 0 & + & + & + & 352 & $\begin{array}{c}9 \\
(-)\end{array}$ \\
\hline
\end{tabular}

Index: $\mathrm{CO}_{2}$ eq. $=$ Treibhauseffekt, $\mathrm{SO}_{2}$ eq. $=$ Versauerung

Profil: $\mathrm{B}=$ Bauprozess, $\mathrm{R}=$ Relevante Bestandteile; $\mathrm{N}=$ Nutzung, $\mathrm{E}=$ Entsorgung; + = günstig, $0=$ Mittel; $-=$ ungünstig

4.1.3

\section{Folgerungen}

Die vorgestellte Studie bietet die Möglichkeit,

Baukonstruktionen gleicher Funktion und ähnlichen

Gebrauchswertes anhand quantifizierbarer Umweltparameter

für die erste Phase des Lebenszylkluses und qualitativer Kriterien für die späteren Lebensphasen miteinander zu vergleichen. Sie bestätigt und ergänzt damit frühere Untersuchungen mit ähnlicher Zielsetzung (Richter, Sell 1992). Es kann festgestellt werden, daß Holzkonstruktionen bei der Index-Beurteilung insgesamt positiv abschneiden, vor allem aufgrund ihrer Vorteile bei der Herstellungsenergie und der Kohlendioxidneutralität. Bei der qualitativen Profil-Beurteilung dagegen werden die Holzkonstruktionen eher als mittelmäßig eingestuft. Dies ist durch die mitverwendeten Stoffe und Produkte (im Kriterium relevante Bestandteile) sowie durch die zusammensetzung der ausgewählten konstruktionen bedingt. Es sollte versucht werden, auch die Profilkriterien in Fallstudien quantitativ zu erfassen und auf eine realistischere Grundlage zu stellen.

\section{2}

\section{Freileitungsmasten}

Um auch imprägnierte Holzteile in Ökobilanzen zu bewerten und den Anforderungen an eine Einbeziehung des gesamten Lebenszyklus zu genügen, wurde eine Studie mit dem Bilanzobjekt „Leitungsmast" durchgeführt (Künniger, Richter 1995). Die imprägnierte Holzstange steht in einigen Ländern aufgrund der Holzschutzdiskussion unter einem steigenden Umweltdruck, und es sollte geprüft werden, wie Holzmast in einer Lebenszyklusanalyse gegenüber den alternativen Tragwerken Stahl und Beton einzuordnen sind.

\subsection{1}

\section{Randbedingungen}

Die zugrundegelegte Ausführung und Bemessung der Masten und Strecken beruht auf realistischen Angaben. Da

Einzeltragwerke hinsichtlich der funktionellen Vergleichbarkeit nicht als bestgeeignet angesehen wurden (unterschiedliche Tragfähigkeiten und Spitzenzüge und damit unterschiedliche Mastzahl pro Streckenlänge), wurden als Basis der 
Gegenüberstellungen ganze Streckennetze zweier Spannungsebenen (0.4- und 20-kV Nieder- und Mittelspannungsleitung) aus salzimprägniertem Rundholz, armiertem Beton und korrosionsgeschütztem Rundstahl herangezogen. Sämtliche mit der Bereitstellung der Tragwerke verbundenen Stoff- und Energieflüsse inklusive der durch sie ausgelösten Emissionen wurden ermittelt. Die Aufwendungen für die Bereitstellung von Infrastruktur, Maschinen und Gebäuden sind nicht einbezogen. Die Rohstoff- $/$ Ressourcengewinnung wurde entsprechend der Herkunft der Materialien bewertet. Für die Mastenherstellung wurden die Verhältnisse der wichtigsten Schweizer Produzenten erfasst, die eine gute bis fortschrittliche Umwelttechnologie aufweisen. Die Bemessung der Masten, die Einbau-, Nachpllege- sowie Entsorgungs- und Deponievorschriften beruhen auf den nationalen Regulierungen.

Die Einwirkungen der Bereitstellung der Elektrizität wurden anhand des europäischen Strommodells UCPTE berechnet. Allerdings sind auch Berechnungen nach dem Schweizer Strom-Mix erfolgt, der infolge des hohen Anteils an Energie aus Wasserkraftwerken ökologisch bedeutend günstiger ist. Jedoch ergaben sich aus dem gewählten Strommodell keine relativen Unterschiede zwischen den Materialvarianten, so daß hier nur die UCPTE-Daten vorgestellt werden.

Alle Berechnungen sind auf eine 60jährige Nutzungsdauer der Masten bezogen. Weil Holzmasten eine statistische Lebensdauer von ca. 30 Jahren aufweisen, ist bei allen Holztragwerken eine einmalige Auswechslung berücksichtigt.

Die Berücksichtigung der Verwertung basiert auf folgenden Annahmen:

Holzmasten: 90\% Verbrennung in einer Kehrichtverbrennungsanlage mit effizienter Rauchgasreinigung (Energierückfluß gutgeschrieben; Asche und Filterstäube werden in Sondermülldeponie endgelagert). 10\% der Masten werden als Zaunmaterial etc. weiterverwendet und werden im Boden abgebaut (die Anteile an Holzschutzmittel sind als Bodenbelastung bewertet).

Betonmasten: $80 \%$ Weiterverwertung als Befestigungselemente im Tief-, Straßen-, Landschafts- oder Wasserbau (keine weiteren Umwelteinwirkungen berücksichtigt). $20 \%$ gehen in eine Inertstoffdeponie.

Rundstahlmasten: 100\% Recycling (nur Transporte zum Altstoffhändler werden berücksichtigt).

\subsection{2}

\section{Ergebnisse}

Die Ergebnisse sind am Beispiel der 0.4-kV-Regelleitung dargestellt (Bilder 4, 5a,b). Die Profilanalyse belegt für keinen Mastentyp Vor- oder Nachteile in allen betrachteten Umwelteffekten. Dennoch sind deutliche Unterschiede auszumachen. Die Stahlleitung hat in acht von neun Evaluationsgrößen die höchsten Belastungen, hauptsächlich verursacht durch die Einwirkungen bei der

Primärstahlproduktion und der Bereitstellung der Energieträger. Die Tragwerke aus Rundholz benötigen nur geringfügig weniger Gesamtenergie als die Betonmasten, jedoch basiert nur ein geringer Anteil dieser Energie auf fossilen Energieträgern. Daher trägt die Holzleitung auch deutlich weniger zum Treibhauseffekt bei, und ihr Beitrag zur Versauerung und Überdüngung ist bedeutend geringer verglichen mit Stahl und Beton. Das Potential für Ökotoxizität ist bei Holzmasten im Vergleich zu Beton allerdings wesentlich höher, als Konsequenz der Holzschutzmittelauslaugung über die Lebensdauer von 2 mal 30 Jahren und der Zinkkorrosion der Stahlzubehörteile der Holzmasten. Vom stehenden

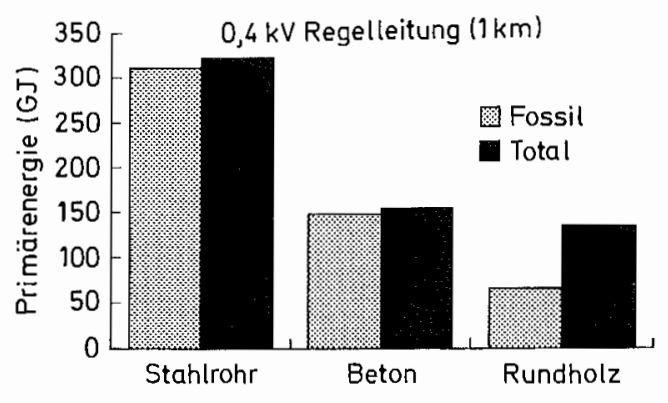

Bild 4. Kumulierter Primärenergieinhalt der 0,4-kV-Niederspannungsleitung

Fig. 4. Cumulated primary energy consumption of the $0,4-\mathrm{kV}$ distribution line
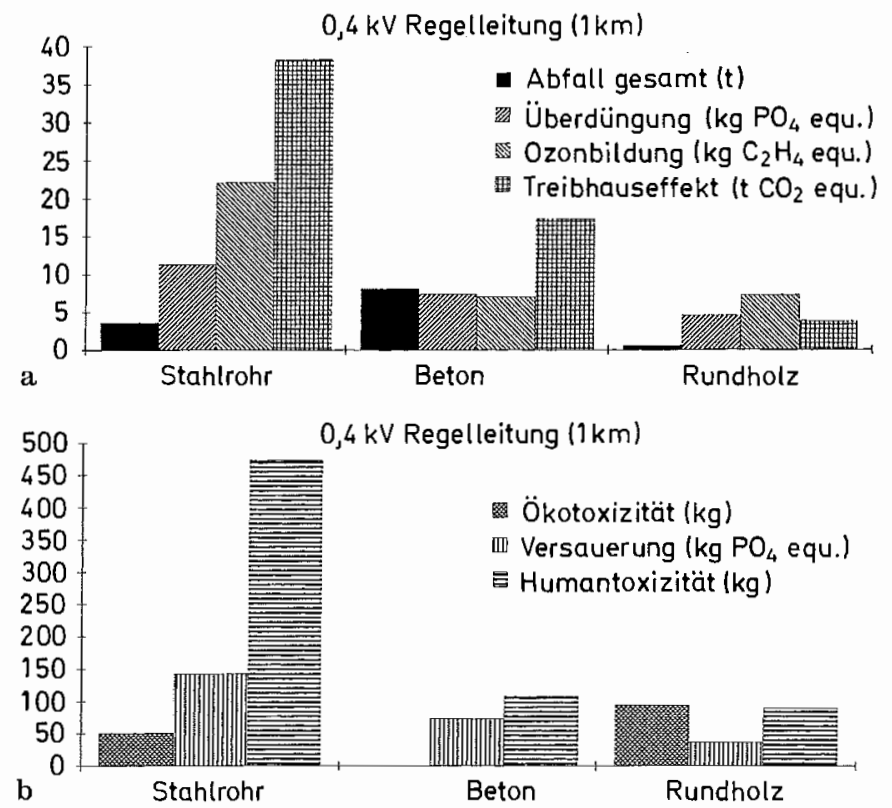

Bild 5. a Gesamtabfallmenge sowie Wirkungspotentiale zu Überdüngung, Ozonbildung und Treibhauseffekt er $0.4-\mathrm{kV}$ Niederspannungsleitung b Wirkungspotentiale zu Ökotoxizität, Versauerung und Humantoxizität der $0,4-\mathrm{kV}$-Niederspannungsleitung Fig. 5. a Waste volume and impact factors for nutrification, photochemical ozone creation and global warming of the $0.4-\mathrm{kV}$ distribution line $\mathbf{b}$ Impact factors for ecotoxicity, acidification and human toxicity of the $0.4-\mathrm{kV}$ distribution line

Betonmasten gehen keine Umwelteinwirkungen an die Umgebung aus, jedoch verursachen sie nach dem Ausbau den höchsten Anteil an festen Abfällen. Der Trend dieser Ergebnisse bestätigt sich bei der Analyse der Einzelmasten und der 20-kV-Mittelspan- nungsleitung. Auch eine Wirtschaftlichkeitsanalyse, die auf den für die Ökobilanz zusammengestellten Sachbilanzdaten beruht, begünstigt eindeutig die Holzstrecken gegenüber den alternativen Bautypen (Künniger et al. 1995).

\subsection{3}

\section{Folgerungen}

Es ist nicht ohne genauere Prüfung möglich, aus dieser Fallstudie auf andere Anwendungen imprägnierter Holzprodukte zu schließen. Wie auch in anderen vergleichenden Ökobilanzen werden markante Umweltvorteile für Holzbauteile zahlenmäßig belegbar, insbesondere beim Energieverbrauch bis zur Herstellung, beim Treibhauseffekt, beim Abfallvolumen. Die Umweltwirkungen der Schutzmittelbehandlung kommen im Bewertungsparameter Ökotoxizität zum Ausdruck, wo die Holzleitung deutlich 
schlechter im Vergleich zur Betonvariante abschneidet. Die Ökobilanz kann nicht entscheiden, wie dieser Sachverhalt im Gesamtzusammenhang zu werten ist. Hier sind erweiterte Modellbetrachtungen nötig, die auch das potentielle Gefährungs- und Schadensrisikopotential mitberücksichtigen müssen.

Auch ohne diese Kenntnisse jedoch können die folgenden Ansätze zur Prozessoptimierung angeregt werden:

- bei Holzmasten: Verbesserung der Fixierungseigenschaften der Schutzmittel, Verwendung korrosionsgeschützter Stahlteile

- bei Beton: Einsatz höherer Anteile erneuerbarer Energieträger bei der Zementbrennung sowie bessere Recyclierungsverfahren zur Verminderung der Abfallmassen - bei Stahl: Höherer Einsatz von Recyclingstahl und eine generelle Materialeinsparung (Gittermaste).

\section{5}

\section{Ausblick}

Ökobilanzen können dazu beitragen, die Umweltwirkungen durch Produkte und Prozesse transparent und bewertbar zu machen. Allerdings steckt die hierzu entwickelte Methodik noch in den Anfängen und unterliegt kurzfristigen Anpassungen und Modifikationen. Es dürfte in den kommenden Jahren gelingen, die Vorgehensweise bis und mit der Bewertungsphase zu festigen und $\mathrm{zu}$ weitgehend anerkannten Richtlinien $\mathrm{zu}$ kommen. Zur Beurteilung der heute noch wenig transparenten Auswirkungen von Stoffen auf die Human- und Ökotoxikologie sind weitere Methodenansätze zu prüfen, damit diese für eine Umweltbewertung wichtigen Aspekte transparenter werden.

Die Aussagefähigkeit vergleichender Ökobilanzen steigt mit der Qualität der benutzten Daten und der Unabhängigkeit, Neutralität und Transparenz der Studien. Es ist in den kommenden Jahren damit zu rechnen, daß die Verfügbarkeit und die Qualität des Daten zunehmen. Dazu können die Unternehmen der Holzindustrie durch eine Offenlegung von Sachbilanzdaten über ihre Produkte und Prozesse allerdings entscheidend beitragen.

Bisher vorliegende, vergleichende Ökobilanzen weisen Vorteile für Produkte aus einheimischem Holz auf, obwohl die Beurteilungen noch selten über den gesamten Lebenszyklus durchgeführt werden. Die positive Stellung des Holzes wird vor allem durch den geringen Einsatz von fossilen

Primärenergieträgern bei Holzernte und Verarbeitung und den geschlossenen $\mathrm{CO}_{2}$-Kreislauf der Rohstoffs hervorgerufen. Die in der Regel geringe Masse von Holzkonstruktionen und die kleinen, nach einer thermischen Nutzung am Ende der stofflichen Nutzung zu entsorgenden Massen (in der Regel Stäube und Aschen) sind weitere Kriterien, bei denen Holz seine vorteilhafte Stellung gegenüber anderen Materialien zum Ausdruck bringt. Dabei finden wesentliche Umwelteigenschaften der Waldwirtschaft bislang noch keine quantitative Berücksichtigung in der Sachbilanzen.

Holzprodukte werden ihre Stellung im Vergleich zu Produkten aus alternativen Materialien vor allem dann behaupten und ausbauen, wenn sie auch qualitativ und preislich konkurrenzfähig sind. Insbesondere für die Gewährleistung einer hochstehenden Funktionstüchtigkeit und langen Lebensdauer ist es für viele Anwendungen nötig, Holz in Kombination mit Hilfs- und Zusatzstoffen (Kleb-, Anstrichstoffe, Schutzmittel, Beschichtungen etc.) zu verarbeiten. Dadurch kann das ursprüngliche Ökoprofil des Hauptmaterials beeinträchtigt werden. Materialkombinationen und Veredelungsschritte müssen vor dem Hintergrund ihrer ökologischen Konsequenzen sorgfältig geplant werden. Eine spätere Trennbarkeit sollte vorgesehen, eine anforderungsgerechte thermische Entsorgung von holzhaltigen Komposites darf nicht verhindert werden.

Es sollte gelingen, das herausragende ökologische Potential von Holz und Holzprodukten in Zukunft noch deutlicher darzustellen. Dafür ist die Zusammenarbeit und Unterstützung aller in der Forst- und Holzkette eingebundenen

Entscheidungsträger notwendig. Mit abgesicherten und glaubwürdig erhobenen Sachbilanzen kann sich Holz in vergleichenden Ökobilanzen mit alternativen Materialien messen und seine ökologischen Stärken zum Ausdruck bringen.

\section{6}

\section{Literatur}

AFB (ed.) 1995: Hochbaukonstruktionen nach ökologischen Gesichtspunkten. erfa info 5/95. Erfahrungsaustausch und Bauökologie. Amt für Bundesbauten, Bern. $14 \mathrm{~S}$

AFB 1995: Nutzungszeiten von Gebäuden und Bauteilen. Amt für Bundesbauten, Bern. $12 \mathrm{~S}$

Braunschweig, A.; Müller-Wenk, R. 1993: Ökobilanzen für Unternehmungen. Eine Wegleitung für die Praxis. Verlag Paul Haupt Bern. $224 \mathrm{~S}$

Gilgen, P. W.; Bieri, E.; Bischoff, E.; Gresch, P.; Zürcher, M. 1993: Betriebliches Umwelt-Informationssystem (BUTS). WF Studie 3, $66 \mathrm{~S}$ CORRIM (Committee on Renewable Resources for Industrial Manufacturing) 1976. Renewable resources for industrial materials. National Academy of Science, Washington D.C., 35p

Heijungs, R.; Guinée, J. B. et al. 1992: Environmental Life Cycle Assessment of Products. Guide and Background. Centrum for Milieukunde, Leiden

FLUMROC 1995: Erster Flumroc-Ökologiebericht. Flumroc AG, Flums, $18 \mathrm{~S}$

ISO (International Organization for Standardization) 1995: 'Technical documents and working drafts of Technical Committee 207, Subcommittee 5: Life Cycle Assessment

Isover 1995: Umweltrapport 1994: Isover AG, Lucens, $17 \mathrm{~S}$ Kasser, U.; Pöll, M. 1995: Graue Energie von Baustoffen. Büro für Umweltchemie, Zürich, $75 \mathrm{~S}$

Kohler, N. A. 1986: Analyse énergetique de la construction de l'utilisation et de la demolition de bâtiments. These No. 623, EPFL, Lausanne, $190 \mathrm{~S}$

Kohler, N. A. (ed.) 1994: Energie- und Stoffflussbilanzen von Gebäuden während ihrer Lebensdauer. Schlussbericht BEW Forschungsprojekt,

EPFL-LESO/ifib Universität Karlsruhe, Karlsruhe. 9 Kapitel + Anhänge Künniger, T.; Richter, K. 1995: Ökologischer Vergleich von Freileitungsmasten aus imprägniertem Holz, armierten Beton und korrosionsgeschütztem Stahl. Forschungsbericht EMPA Abt. Holz, $233 \mathrm{~S}$

Künniger, T.; Richter, K.; Dauwalder, R. 1995: Ölkobilanz über Tragwerke von Mittel- und Niederspannungsfreileitungen. Bulletin SEV/VSE 24, 45-51

Ressel, J. 1986: Energieanalysen der Holzwirtschaft der Bundesrepublik Deutschland. BMFT-FBT 86/184, $161 \mathrm{~S}$

Richter, K.; Sell, J. 1992: Ökobilanzen von Baustoffen und Bauprodukten aus Holz. Zusammenfassung erster Ergebnisse. Forschungs- und Arbeitsbericht der EMPA-Abt. Holz Nr. 115/24, 33 S Richter, K. 1994: Ansätze zur Ölkobilanzierung von Werkstoffen unter besonderer Berïcksichtigung des Werkstoffs Holz und der schweizerischen Methodendiskussion. In: FGU Berlin (ed.) Ökobilanzen. Tagungsbroschüre Umwelttechnologieforum Berlin, 10. Seminar, $163-169$

Richter, K. 1995: Ökoprofil von Holz- und Holzwerkstoffe.

Tagungsband des 27 SAH Fortbildungskurs 'Innenräume in Holz', S. $119-126$

Rubik, F.; Teichert, V. 1995: Ökologische Produktpolitik. Stuttgart, C.E. Poeschel

SETAC (Society for Environmental Toxicology and Chemistry) 1993: Guidelines for Life-Cycle Assessment: A 'Code of Practice' 1. ed., Brussels

SIA (ed) 1995: Hochbaukonstruktionen nach ökologischen

Gesichtspunkten. Dokumentation D0123, Bearbeiter Intep AG und P. Steiger, Zürich, 13 S. und Anhänge

Weibel, Th.; Stritz, A. 1995: Ökoinventare und Wirkungsbilanzen von Baumaterialien. ETH Zürich, ESU-Reihe 1/95, 28 S. und Anhänge 
